\title{
Continuation-based semantics for Conventional Implicatures: The case of Japanese benefactives
}

\author{
Yusuke Kubota and Wataru Uegaki \\ The Ohio State University and the University of Tokyo
}

\section{Introduction}

This paper proposes a novel, fully compositional analysis of conventional implicatures (CIs) (in the sense of Potts (2005)) in terms of the technique of continuations (Barker 2002, 2004). The paper has both an empirical and a theoretical goal. The empirical goal is to point out the existence of phenomena (including the Japanese benefactive predicate morau) in which a certain expression contributes meanings both at the level of ordinary assertion and at the level of CIs. Such cases are predicted to be nonexistent in natural language by an influential theory of CIs developed by Potts (2005). The theoretical goal is to propose an alternative formal treatment of CIs that overcomes this empirical shortcoming of Potts's theory. As we will show below, by employing the technique of continuations, a theoretically simpler treatment of CIs becomes possible, which simultaneously overcomes the above empirical problem of Potts's original formulation of CI logic.

\section{Potts's (2005) theory of Conventional Implicatures}

\subsection{What are CIs?}

In Potts (2005), CIs are characterized as linguistically triggered noncancelable entailments that have truth conditions independent of ordinary entailments (or, atissue meanings, to use Potts's terminology). ${ }^{1}$ A typical case of CI-triggers is the class of parenthetical expressions (supplements in Potts's terminology) including the nonrestrictive relative clause in (1):

(1) John, who is smart, passed the exam.

Of the two entailments of this sentence, 'John passed the exam' and 'John is smart', the latter, which is contributed by the parenthetical, is a CI.

Potts discusses several properties of CIs that distinguish them from both at-issue meanings and presuppositions. We review here the most important and

\footnotetext{
${ }^{1}$ We do not include speaker-orientation as a definition of CI, since this criterion does not seem to be entirely exception-free; as Amaral et al. (2007) point out, there are cases where supplements, a typical case of CI triggers, generate CIs that are attributable to attitude-holders other than the speaker (for specific examples, see Amaral et al. (2007); cf. also Wang et al. (2005) and Harris and Potts (to appear)).
} 
clearest criteria for each. First, CIs can be distinguished from at-issue meanings in that they do not fall under the scope of truth-functional operators, or, the so-called presupposition holes, such as negation, question, modals and the antecedent of conditionals. For example, (2) shows that the meaning contribution of the supplement in (1) projects beyond the scope of the question operator. B's response is infelicitous since it takes the entailment contributed by the supplement as part of what is being asked by A's utterance.

(2) A:Did John, who is smart, pass the exam?

B:\#No, he isn't smart although he passed the exam.

Second, CIs are different from presuppositions in that the truth value of the at-issue content can be determined even if the CI is false (whereas presupposition failure typically leads to uninterpretability modulo accommodation). In our parenthetical example, this is in fact the case since the truth of the claim made in (1) (i.e. whether John passed the exam) can be determined even if the contribution of the parenthetical (i.e. the proposition 'John is smart') is known to be clearly false, as evidenced in (3). By contrast, such is not the case with presuppositions; although presupposition is a notoriously elusive notion, it is one of its defining characteristics that presupposition failure leads to a situation in which the sentence simply lacks a truth value, in other words, cannot (even) be true or false. The infelicity of B's response in (4) (which is in stark contrast to the relative acceptability of (3)) shows the relevant point.

(3) A:Did John, who is smart, pass the exam?

B:Yes, he did pass the exam. But just so you know, he isn't actually smart.
A:Did John stop smoking?
(presupposition)

B:\#Yes. But just so you know, he hasn't been a smoker to begin with.

\subsection{Multidimensional logic for CIs: $\mathscr{L}_{\mathrm{CI}}$}

Based on the above pretheoretical characterization of CIs as entailments that are independent of at-issue meanings, Potts (2005) develops a theory of CIs whose central characteristic is its multidimensionality. CIs and at-issue meanings are formally treated as different kinds of objects belonging to mutually exclusive series of semantic types, specifically, at-issue types and CI types, as defined in (5).

a. at-issue type: $e^{a}, t^{a}, s^{a},\left\langle\sigma^{a}, \tau^{a}\right\rangle \quad$ (with $\sigma^{a}$ and $\tau^{a}$ at-issue types)

b. CI type: $e^{c}, t^{c}, s^{c},\left\langle\sigma^{a}, \tau^{c}\right\rangle \quad$ (with $\sigma^{a}$ an at-issue type and $\tau^{c}$ a CI type)

With this type distinction, Potts defines separate semantic rules such as the ones in (6) for calculating at-issue meanings and CIs compositionally. ${ }^{2}$

\footnotetext{
${ }^{2}$ The dashed box indicates that the element enclosed in it is optional.
} 
(6)

a. at-issue application

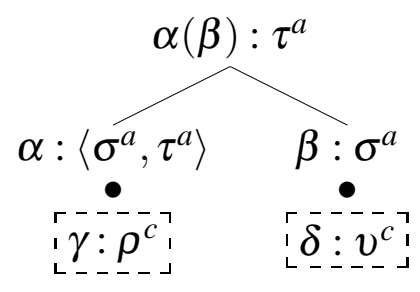

b. CI application

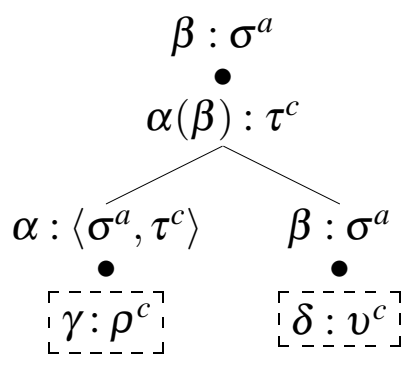

Here, $\bullet$ is a metalogical symbol for separating CIs from at-issue contents. (6a) is basically the ordinary function application rule affecting only the at-issue meaning at each node. CI application (6b), on the other hand, is an identity function as far as the at-issue meaning is concerned. But this latter rule has the additional effect of applying a CI functor $\alpha$ (of type $\left\langle\sigma^{a}, \tau^{c}\right\rangle$ ) to its argument $\beta$ (of type $\sigma^{a}$ ) to calculate the $\mathrm{CI}$ of the larger expression. Note especially the ways in which these rules crucially make reference to the semantic type distinction between the at-issue and CI types to achieve the intended semantic effects.

With these rules, the parenthetical in example (1) can be analyzed as in the diagram shown in (7), which Potts calls the parsetree of the sentence.

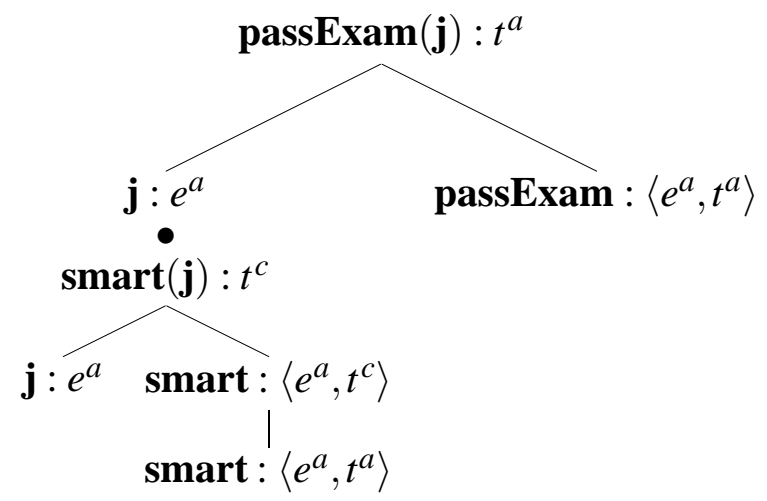

The parenthetical, being of a CI functor type $\left\langle e^{a}, t^{c}\right\rangle$, applies to the subject NP John to generate a CI ('smart (j)'), but does not affect the at-issue content of the modified NP. Thus, the subject NP modified by the parenthetical can combine with the matrix VP to form a sentence that has the expected at-issue entailment.

Once the parsetree for the whole sentence is constructed, the at-issue and CI meanings are separately retrieved from the tree in accordance with a convention called 'parsetree interpretation', which dictates that the at-issue meaning of the root node be the at-issue meaning of the whole sentence and that the conjunction of all of the type $t^{c}$ meanings (if there are any) retrieved from any node in the whole tree be the CI meaning of the whole sentence. From this, we can see that the parsetree is a representational device in Potts's theory in the sense that it constitutes an ineliminable theoretical construct for obtaining the final semantic interpretation of the sentence.

As should be clear from the above summary, Potts's system neatly captures the independence of CI meanings from at-issue meanings by means of the type 
distinction between them. This type distinction has several nontrivial consequences. We focus here on the most important one for our purposes: ${ }^{3}$

No lexical item contributes both an at-issue and a CI meaning. (Potts 2005: 48)

This consequence follows in Potts's system because every lexical item has to be well-typed, that is, it has to belong to either an at-issue type (in which case, it contributes an at-issue meaning only), or a CI type (in which case, it contributes a CI meaning only, possibly by taking some other expression as an argument). However, this prediction of $\mathscr{L}_{\mathrm{CI}}$ turns out to be empirically too strong: there are cases where a single lexical item makes semantic contributions both at the CI and at-issue levels simultaneously, as we will see in the following section.

\section{Japanese benefactives: 'Mixed' CI contributions}

As an example that goes against the generalization stated in (8), we consider here (and analyze in section 4) the Japanese benefactive verb morau, as exemplified in the following sentence: 4

$$
\begin{aligned}
& \text { Taroo-ga Hanako-ni piano-o hii-te morat-ta. } \\
& \text { Taro-NOM Hanako-DAT piano-ACC play BENEF-PAST } \\
& \text { at-issue: 'Taro had Hanako play the piano.' } \\
& \text { CI: 'Hanako's playing the piano was for the benefit of Taro.' }
\end{aligned}
$$

In (9), the benefactive verb morau embeds another verb (hii-te 'play') and identifies its own dative object (Hanako-ni) as the logical subject of the embedded verb. The sentence as a whole describes an event in which the matrix subject is involved

\footnotetext{
${ }^{3}$ Apart from the empirical problem discussed in the text, the semantic type distinction between at-issue and CI meanings is ontologically somewhat dubious as well. For example, $\left\langle e^{a}, t^{a}\right\rangle$ and $\left\langle e^{a}, t^{c}\right\rangle$ are formally distinct types despite the fact that expressions belonging to each denote exactly the same kind of model theoretic objects, that is, properties of individuals. (Morzycki (2008: 110, footnote 10) makes the same observation.)

${ }^{4}$ Other similar cases include: the T/V distinction of personal pronouns in some European languages (Horn 2007); Japanese emotive adverbs sekkaku and yokumo (McCready 2009); Japanese scalar reversal adverbs kaette and yoppodo (Sawada to appear); intensifiers like ur (German), totally (English) and zenzen (Japanese) (McCready and Schwager 2009). Japanese adversative passive and suppletive honorific forms of certain verbs (e.g. mesiagaru) can be added to this list.

However, a care needs to be taken in adducing these cases as counterevidence for Potts's formulation of his CI logic, since many (but not all) of these cases seem to involve expressive CIs, for which it is not obvious whether the kind of multidimensional truth-conditional treatment (which characterizes Potts's original system as well as our reformulation of it) is suitable in the first place (see Bach (2006) for a pointed discussion on this issue). We leave for future study to identify which, if any, of the phenomena listed here (or any other phenomena) will constitute true counterevidence for Potts's theory (i.e. count as 'mixed', non-expressive CI contributors). The Japanese benefactive verb discussed in the text is clearly not an expressive, since the benefactive meaning associated with it does not necessarily reflect an attitude on the part of the speaker (e.g., when embedded under propositional attitude predicates, the $\mathrm{CI}$ could be ascribed to the attitude holder of the predicate).
} 
(in some way or other) in the embedded (logical) subject's action described by the embedded verb, which, in turn, is a beneficial state of affair for the matrix subject. ${ }^{5}$ What is crucial here is that the benefactive meaning is a CI whereas the 'involvedness' meaning is an at-issue meaning.

The CI-hood of the benefactive meaning can be supported in the same way as in the case of an English parenthetical expression. First, the benefactive meaning projects out of presupposition holes such as negation, as shown in (10), which indicates that it is not an at-issue meaning.

(10) Taroo-wa Hanako-ni piano-o hii-te moraw-anakat-ta. \#Toiunomo, Taro-TOP Hanako-DAT piano-ACC play BENEF-NEG-PAST for [Taroo-ga Hanako-ni piano-o hik-ase-ta]-no-wa jibun-no Taro-NOM Hanako-DAT piano-ACC play-cause-PAST-COMP-TOP self-GEN tame de-wa nakat-ta kara. sake TOP NEG-PAST because intended: 'Taro didn't have Hanako play the piano for him. For it was not for the benefit of himself that he made her play the piano.'

In (10), the negative morpheme attaching to the matrix benefactive verb morau cannot target the benefactive meaning contributed by morau, as evidenced by the infelicity of the second sentence, which is intended to force such an interpretation.

Second, the at-issue content can be judged as true even when the benefactive meaning is false, as shown in the following discourse, from which we can see that

\footnotetext{
${ }^{5}$ The at-issue meaning of morau is somewhat difficult to delineate. Typically, the 'involvedness' relation is instantiated as a causative relation (that is, the matrix subject causes the embedded subject to do the action described by the embedded verb). However, there are examples like the following, which suggest that such a causative relation is not a semantic entailment of the benefactive verb:

(i) Taroo-ga Hanako-ni kizetu-si-teiru tokoro-o okosi-te morat-ta. Taro-NOM Hanako-DAT be.unconscious NMLZ-ACC wake.up BENEF-PAST 'Hanako woke Taro up while Taro was unconscious, which was beneficial for Taro.'
}

One might think, on the basis of examples such as (i), that morau can be analyzed as involving no at-issue meaning at all. Such an analysis, however, is problematic for two reasons. First, adding the benefactive verb changes the argument structure of the original verb (specifically, the original subject becomes a dative argument and an extra subject that does not bear any semantic relation to the original verb is newly added). This argument structure change cannot be accounted for properly if one assumes that the contribution of morau is completely vacuous at the at-issue level.

Second, the benefactive verb can cooccur with adverbials which can only be understood as modifying the at-issue meaning of the benefactive verb. For instance, in (ii), the adverb muriyari 'forcefully' is interpreted as modifying neither the benefactive meaning nor the meaning of the embedded clause, but rather, as predicating of the way in which Taro is involved in (or, in this particular case, causing) the event of Hanako's playing the piano.

(ii) Taroo-ga Hanako-ni muriyari piano-o hii-te morat-ta. Taro-NOM Hanako-DAT forcefully piano-ACC play BENEF-PAST at-issue: 'Taro forcefully made Hanako play the piano.' CI: 'Hanako's playing the piano was for the benefit of Taro.'

Such examples cannot be accounted for if we do not analyze morau as involving an at-issue meaning. 
the benefactive meaning in question is not a presupposition.
A:Taroo-wa Hanako-ni piano-o hii-te morat-ta-no?
Taro-TOP Hanako-DAT piano-ACC play BENEF-PAST-Q
'Did Taro have Hanako play the piano for him?'
B:Hai, tasikani Taroo-wa Hanako-ni piano-o hik-ase-ta-yo.
yes, certainly Taro-TOP Hanako-DAT piano-ACC play-CAUSE-PAST-PRT
Demo, sore-wa Taroo jisin-no tame de-wa nai kedo.
but that-TOP Taro self-GEN sake TOP NEG though
'Yes, he did make her play the piano. But it wasn't for the benefit of himself that he did so.'

In this example, B affirms A's question by making a stronger assertion that the manner in which Taro was involved in Hanako's playing the piano was in terms of a causative relation, while explicitly negating the benefactive meaning contributed by morau. The felicity of B's response here shows the independence of the at-issue, involvedness meaning from the benefactive meaning of morau.

Given the above observations, we conclude that the benefactive meaning associated with morau is a CI. This means that morau contributes both the at-issue involvedness meaning and the CI benefactive meaning at the same time. (Following McCready (2009), we call such expressions 'mixed' type CI contributors.) As such, there is no way in $\mathscr{L}_{\mathrm{CI}}$ to account for the behavior of this expression, since it cannot be assigned an appropriate lexical entry in the first place (specifically, it is neither an at-issue type expression nor a CI-type expression). The question that then arises is whether it would be possible to give up the strict, type-driven multidimensional architecture of $\mathscr{L}_{\mathrm{CI}}$ (which is the cause of the problem) but still retain its original insight wherein the independence of the at-issue and CI meanings is captured. In the next section, we show that the technique of continuations, as applied to certain linguistic phenomena in the recent literature (cf, e.g., Barker (2004) for an overview), provides exactly the kind of mechanism that we want here. ${ }^{6}$

\section{Continuation-based semantics for CIs}

\subsection{Continuations for natural language semantics}

Before presenting our compositional theory of CIs, we briefly review the notion of continuations. Our exposition here is mostly based on Barker and Shan (2008). For a more detailed (linguistically-oriented) discussion of foundational issues and other applications, see, e.g., Barker (2002, 2004) and Shan and Barker (2006).

\footnotetext{
${ }^{6}$ An alternative (and more conservative) approach is to augment $\mathscr{L}_{\mathrm{CI}}$ with more types and more rules, as is proposed, for example, by McCready (2009). The reason that we opt away from such a solution is that (i) such a modification introduces a significant complication to the overall theory (especially, in the type system) and that (ii) we think that the type system is not the right place to encode the distinction between at-issue and CI meanings (cf. footnote 3 ).
} 
The notion of continuations has been originally developed in theoretical computer science for handling evaluation order of a program in computation (cf., e.g., Plotkin 1975, Reynolds 1993). Recently, Barker and Shan (Barker 2002, Shan and Barker 2006, Barker and Shan 2008, Barker 2009) have proposed several applications of this technique to some of the recalcitrant problems in natural language semantics, including quantifier scope (Barker 2002), weak crossover and superiority (Shan and Barker 2006), donkey anaphora (Barker and Shan 2008) and reconstruction (Barker 2009), providing explicit and fully compositional fragments dealing with these issues. While there are several different ways for implementing the notion of continuations within a formal linguistic theory, in this paper we follow Barker and Shan (2008) and Barker (2009) in adopting a fragment of combinatory categorial grammar with the 'tower notation', a recent notational innovation by Barker and Shan (2008). ${ }^{7}$ The choice of the tower notation here is based on practical considerations: it makes the derivations much easier to read than in earlier formats. In particular, the graphical two-dimensional display of syntactic categories and semantic translations is especially effective for our purposes, since it helps visualize the way in which CIs and at-issue meanings are computed in tandem through the steps of syntactic derivation.

As pointed out above, continuations are a technique for manipulating evaluation order. The most obvious linguistic application of such a notion is for quantifier scope, which can be seen as a typical instance of delayed evaluation in natural language. That is, when a quantifier takes wide scope, the semantic composition has to proceed in such a way that the quantifier does not make any substantial meaning contribution in the local syntactic environment in which it appears, but its quantificational force is integrated with the meaning of the linguistic expression only after a larger context is found that properly licenses its evaluation. ${ }^{8}$ Below, we will illustrate how this works in a concrete fragment of continuation-based grammar.

In our fragment, we write linguistic expressions as triples of phonology, syntactic category and semantic interpretation. A sample lexicon is given in (12). ${ }^{9}$
a. 〈loves; $(\mathrm{NP} \backslash \mathrm{S}) / \mathrm{NP}$; love $\rangle$
b. 〈someone; $\left.\frac{\mathrm{S} \mid \mathrm{S}}{\mathrm{NP}} ; \frac{\exists x \cdot[]}{x}\right\rangle$
c. $\left\langle\right.$ everyone; $\left.\frac{\mathrm{S} \mid \mathrm{S}}{\mathrm{NP}} ; \frac{\forall x \cdot[]}{x}\right\rangle$

The lexical entry for loves is unexceptional. The entries for the quantifiers $(12 \mathrm{~b}, \mathrm{c})$, on the other hand, involve an additional continuation level, written above the line in the tower notation. (We say that such expressions are 'continuized'.) The syntactic

\footnotetext{
${ }^{7}$ For a different implementation, see, e.g., the variant formalized within Type-Logical Grammar by Barker and Shan (2006).

${ }^{8}$ The reader might notice that this informal characterization is reminiscent of Cooper storage (Cooper 1983). This is a good guiding intuition. However, it should be noted that continuations are a much more general notion than Cooper storage and that there are certain linguistic phenomena which the former, but not the latter, can naturally handle, such as parasitic scope, as in the analysis of the internal reading of same by Barker (2007).

${ }^{9}$ Following Barker and Shan (2008), we adopt the 'result-top' notation of slashes. That is, NP $\backslash S$ is a category that is looking for an NP to its left to become an S.
} 
category $\frac{\mathrm{S} \mid \mathrm{S}}{\mathrm{NP}}$ should be read counterclockwise from the bottom: it says that the expression behaves as an NP in its local context and takes scope over S (top right corner) to return an $S$ (top left corner). Correspondingly, in semantics, the term below the line denotes the local meaning contribution and the expression above the line specifies the semantic effect that obtains when the continuized meaning is evaluated in the appropriate larger context. Specifically, the quantifier leaves a (place-holder) variable in the local context and the quantificational operator that binds that variable is stored in the continuation level so that it can later be integrated into the meaning of the whole linguistic expression once some larger expression (of syntactic category S) is created. As will become clear in a moment, this 'storing' of the quantifier meaning in the continuation level is what enables the treatment of quantifier scope in terms of delayed evaluation.

The grammar is equipped with two kinds of rules for dealing with expressions having continuation levels: (i) unary type-shifting rules for adding and collapsing continuation levels and (ii) binary rules for combining linguistic expressions that have continuation levels. We first present the two unary type-shifting rules, Lift and Lower, defined as in (13): ${ }^{10}$
a. $\langle\alpha ; A ; x\rangle \quad \underset{\operatorname{Lift}}{\Rightarrow}\left\langle\alpha ; \frac{\boldsymbol{B} \mid \boldsymbol{B}}{A} ; \frac{[]}{x}\right\rangle$
b. $\left\langle\alpha ; \frac{A \mid \boldsymbol{B}}{\boldsymbol{B}} ; \frac{f[]}{x}\right\rangle \quad \stackrel{\text { Lower }}{\Rightarrow}\langle\alpha ; A ; f[x]\rangle \quad$ (where $B$ is either $\mathrm{S}$ or Assn)

Lift takes a non-continuized expression and adds to it a new continuation level, with a vacuous continuized meaning. This rule is a generalization of the familiar typelift (or type-raising) rule (cf., e.g., Partee and Rooth 1983, Partee 1986) used, for example, to lift a type $e$ proper name to the generalized quantifier type $\langle\langle e, t\rangle, t\rangle$. Lower does the opposite of Lift. That is, it collapses a continuation tower into a flat, non-continuized expression. The semantic effect of Lower is technically an application to an identity function; more intuitively, what effectively happens is that the continuized meaning and the local meaning are merged into a flat, noncontinuized meaning. This rule, again, can be thought of as a generalization of a more familiar rule, specifically, Partee's (1986) type-shifting rule with the same name, which converts a lifted meaning of a proper name back to a type $e$ meaning.

The fragment is further equipped with two binary rules for combining two expressions that have continuation levels to produce a larger expression: ${ }^{11}$

\footnotetext{
${ }^{10}$ Italicized letters $(A, B$, etc.) in rules are variables over syntactic categories; two (or more) occurrences of the same letter in a rule (e.g. $B$ in (13a)) need to be instantiated to the same category.

${ }^{11}$ These rules are named $\mathbf{S}$ since $\mathbf{S}$, corresponds to a rule called 'Scope' in Shan and Barker (2006) and $\mathbf{S}$ is derived from it.
} 


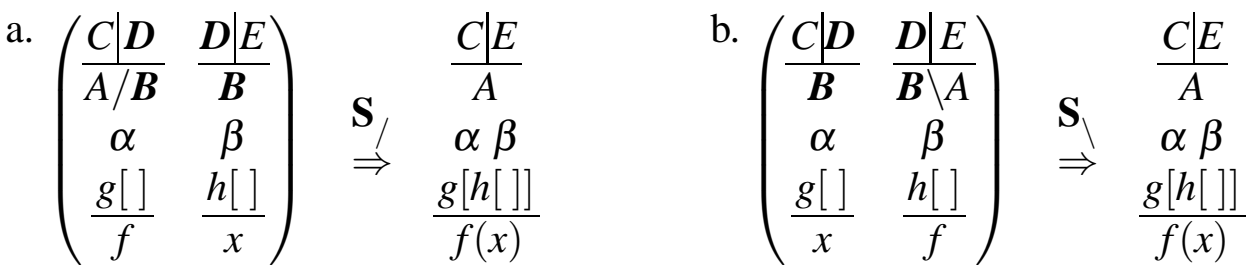

These two binary rules are essentially continuized variants of function application: below the line, we see the familiar effect of function application both in syntax and semantics. What is novel is above the line, where what is involved $(C|D+D| E \Rightarrow$ $C \mid E$ ) can be thought of as a kind of function composition, by which the 'meaning parts' stored in the continuation levels of the daughters are assembled and passed up to the mother. ${ }^{12,13}$

We are now ready to see how quantifier scope is handled in the present fragment. (15) illustrates the derivations for the two readings for the ambiguous sentence Someone loves everyone.

a. (surface scope)

\begin{tabular}{|c|c|c|c|c|c|c|}
\hline $\mathrm{S} \mid \mathrm{S}$ & S & $\mathrm{S} \mid \mathrm{S}$ & & $\mathrm{S} \mid \mathrm{S}$ & & \\
\hline$\overline{\mathrm{NP}}$ & $\overline{(\mathrm{NP} \backslash \mathrm{S}) / \mathrm{NP}}$ & $\overline{\mathrm{NP}}$ & & $S$ & & S \\
\hline $\begin{array}{c}\text { someone } \\
\qquad x .[] \\
\end{array}$ & $\begin{array}{c}\text { loves } \\
{[]} \\
\end{array}$ & $\begin{array}{c}\text { everyone } \\
\forall y .[] \\
\end{array}$ & & $\begin{array}{c}\text { someone } \\
\text { loves everyone } \\
\exists x . \forall y .[\end{array}$ & $\begin{array}{l}\text { Lower } \\
\quad \Rightarrow\end{array}$ & $\begin{array}{c}\text { someone } \\
\text { loves everyone } \\
\exists x \forall y \text { love }(y)(x)\end{array}$ \\
\hline$x$ & love & $y$ & & love $(y)(x)$ & & \\
\hline
\end{tabular}

b. (inverse scope)

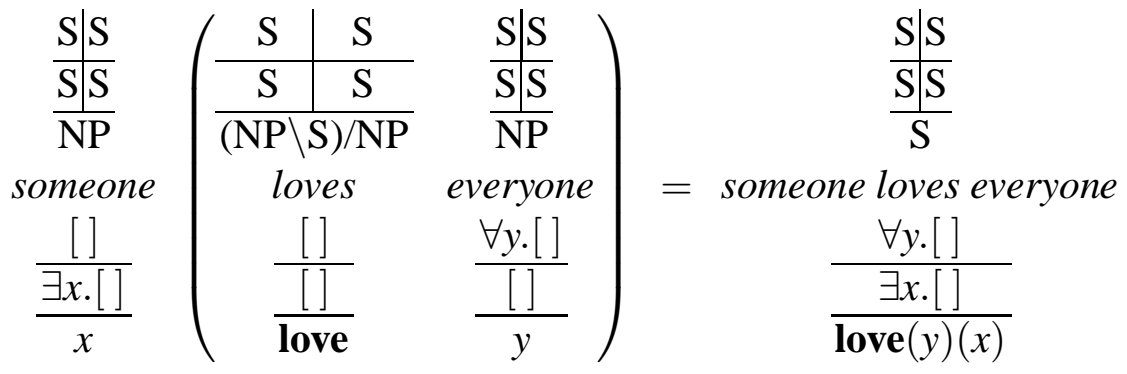

Lower (twice)

$\mathrm{S}$

$\Rightarrow \quad$ someone loves everyone
$\forall y \exists x$ love $(y)(x)$

In the derivation (15a) for the surface scope reading, the verb loves is Lifted so as to match the lexical specifications for the quantifiers in the height of the continuation tower (a prerequisite for the application of $(14 a, b)$ ). Then the three expressions are combined via $(14 \mathrm{a}, \mathrm{b})$, yielding the righthand side of the equation. Note

\footnotetext{
${ }^{12}$ See Shan and Barker (2006) for a more technical exposition of what is exactly involved here.

${ }^{13}$ The continuized application rules, as they are formulated in (14), are actually insufficiently general. The rules should be generalized to handle cases involving multiple continuation levels (cf. Barker and Shan (2008) for a more detailed discussion on this point). In the generalized rule, the 'cancellation' of matching syntactic categories and the composition of the continuized meanings are done separately for each continuation level in the same way as in (14). In what follows, we assume that the fragment is equipped with this generalized versions of continuized application rules.
} 
here the uniquely determined order between the existential and universal quantifiers above the line; this will end up corresponding to the scope relation in the final translation. Once the three expressions are combined with one another, Lower can apply since the category of the top right corner ('input' for the continuation level) and the local syntactic category below the line match. Applying Lower collapses the continuation tower and the ordinary logical translation for the surface scope reading is obtained.

The derivation (15b) for the inverse scope reading is slightly more complicated in that it involves two continuation levels. Note first that, for expressions that are already equipped with a continuation level (such as the quantifiers in $(12 b, c)$ ), there are two distinct ways in which Lift can apply. Specifically, with the quanti-

fiers in $(12 b, c)$, applying Lift to the whole expression of category $\frac{S \mid S}{N P}$ results in the specification for the subject quantifier someone in (15b). By contrast, if the rule targets the lower local syntactic category NP alone, the specification for the object quantifier everyone in (15b) is obtained. Note that the quantificational operators end up in different continuation levels depending on the way in which the rule is applied. The verb loves here undergoes Lift twice so that it matches in tower height with the two quantifiers. The rest is similar to the derivation in (15a). The three expressions are combined by (the generalized variants of) the continuized application rules in (14), which assigns a doubly continuized category and meaning for the whole sentence (righthand side of the equation). This double continuation tower is then collapsed by applying Lower twice, which yields the desired translation for the inverse scope reading. Note that the object quantifier scopes over the subject quantifier in this derivation since its quantificational force is stored at a higher continuation level, which ensures that it is integrated into the meaning of the whole expression only after the subject quantifier takes scope.

To summarize, continuized grammars like the present fragment are equipped with continuation levels that enable one to control the ways in which meaning contributions of linguistic expressions are put together in a flexible manner. The treatment of quantifier scope illustrated above crucially exploits this property in accounting for scope ambiguity as a case that involves partial nondeterminism in the evaluation order of linguistic expressions.

\subsection{Formalizing the theory of CIs}

The technique of continuations outlined above turns out to be perfectly suitable for capturing the behaviors of CIs. As discussed in section 2, CIs are chunks of meanings that do not interact with at-issue meanings; specifically, they do not fall within the scope of truth-functional operators. We can think of this projective behavior of CIs as an instance of delayed evaluation. That is, CI triggers make meaning contributions that are not evaluated in the immediate local contexts; rather, we wait until a larger context is found that licenses the evaluation of these meaning contributions (which is typically the matrix level assertion). 
More specifically, the idea outlined above can be formalized in a continuationbased semantics in the following way. We introduce a distinct continuation level for keeping track of CI meanings in linguistic composition (shown above the line in the 'tower' notation). This continuation level is kept separate from the at-issue meaning by 'locking' it into a special syntactic category called Assn (for 'assertion'). With the help of this special syntactic category, we can guarantee that continuized CIs can be unlocked and integrated with the at-issue meaning only when an appropriate syntactic environment (having Assn as its local category) is found. ${ }^{14}$

\subsubsection{English parentheticals}

We first illustrate the workings of our system through a stepwise presentation of an analysis of English parentheticals, a paradigmatic case of CI trigger. The key component of our analysis is in the following lexical entry for the nonrestrictive relative pronoun who, which produces a two-level continuation tower when combined with the body of the relative clause (of category NP $\backslash S$ ) and the nominal head that the relative clause modifies; in the resultant continuation tower, the CI contribution is specified above the line and the at-issue meaning appears below the line.

$$
\left\langle w h o ;\left(\mathrm{NP} \backslash \frac{\text { Assn } \mid \text { Assn }}{\mathrm{NP}}\right) /(\mathrm{NP} \backslash \mathrm{S}) ; \lambda P \lambda x \cdot \frac{P(x) \wedge[]}{x}\right\rangle
$$

With the lexical entry for who in (16), the derivation for John, who is smart goes as in (17), via successive applications of the ordinary (i.e. non-continuized) function application.

a.

$$
\left(\begin{array}{cc}
\left(\mathrm{NP} \backslash \frac{\text { Assn } \mid \text { Assn }}{\mathrm{NP}}\right) /(\mathrm{NP} \backslash \mathrm{S}) & \mathrm{NP} \backslash \mathrm{S} \\
\text { who } & \text { is smart } \\
\lambda P \lambda x \cdot \frac{P(x) \wedge[]}{x} & \text { smart }
\end{array}\right)=\begin{array}{cc}
\mathrm{NP} \backslash \frac{\text { Assn } \mid \text { Assn }}{\mathrm{NP}} \\
\quad \lambda x \cdot \frac{\operatorname{smart} \text { smart }(x) \wedge[]}{x}
\end{array}
$$

b.

$$
\left(\begin{array}{cc}
\mathrm{NP} & \mathrm{NP} \backslash \frac{\text { Assn } \mid \text { Assn }}{\mathrm{NP}} \\
\text { John } & \text { who is smart } \\
\mathbf{j} & \lambda x \cdot \frac{\mathbf{s m a r t}(x) \wedge[]}{x}
\end{array}\right)=\begin{gathered}
\frac{\text { Assn } \mid \text { Assn }}{\mathrm{NP}} \\
\frac{\text { John, who is smart }}{\mathbf{s m a r t}(\mathbf{j}) \wedge[]}
\end{gathered}
$$

Note here that the at-issue meaning of the relative clause who is smart is an identity function $(\lambda x . x)$ while the meaning stored in the CI dimension is a one place predicate applying to the NP argument that the relative clause attaches to (John in

\footnotetext{
${ }^{14}$ This licensing environment is most typically the matrix level assertion (hence the name Assn for the syntactic category), but the formal system itself allows for more flexibility, which seems empirically necessary given recent discussion (cf., e.g., Amaral et al. 2007, Harris and Potts to appear).
} 
the case of (17b)). We thus obtain the effect of Potts's CI application (6b) without positing any special rule; all that is needed in our system is an appropriate lexical specification for the CI trigger.

Next, we combine the resultant subject (with the relative clause) and the VP passed the exam with rule (14b), as in (18) below. Here, the VP, which does not originally involve a continuation level, is Lifted so that it can combine with the subject, which comes with a continuation level. By this application, the CI is projected to the whole sentence.

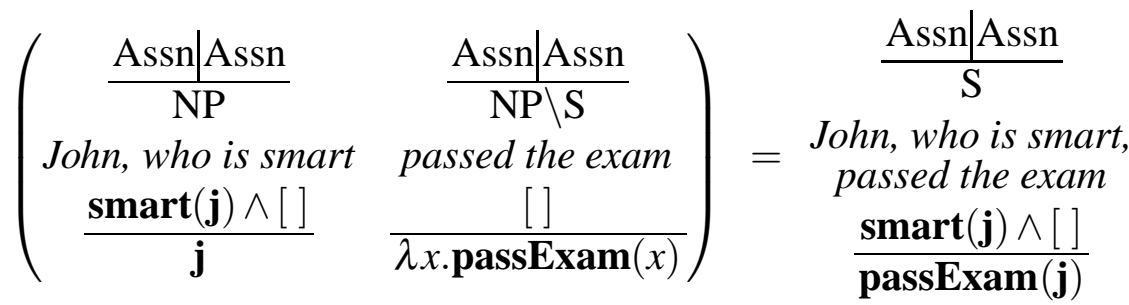

Once the sentence is built up, a unary 'Assertion' rule (19) applies so that the local syntactic category is changed from $S$ to Assn (without any semantic effect). This enables Lower to integrate the CI with the at-issue meaning. These steps are shown in (20). In the final translation, both the at-issue meaning smart (j) and the CI passExam (j) are treated as entailments of the sentence, to the desired effect. ${ }^{15}$

$$
\begin{aligned}
& \langle\alpha ; S ; x\rangle \quad \stackrel{\mathbf{A}}{\Rightarrow}\langle\alpha ; \text { Assn; } x\rangle \\
& \text { Assn|Assn } \\
& \text { Assn Assn }
\end{aligned}
$$

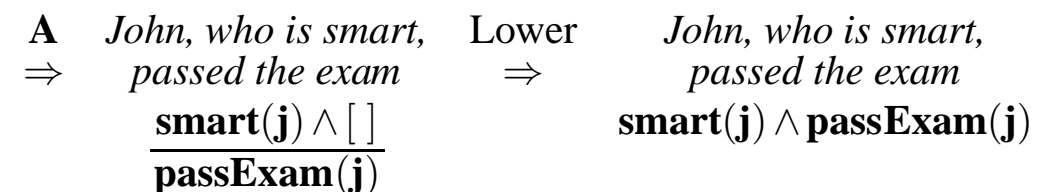

A final note about this derivation: the independence of the CI meaning and the at-issue meaning is ensured by the fact that the CI level is associated with the distinct syntactic category Assn. That is, the projective behavior of CI meanings, i.e., that they don't fall under the scope of truth-functional operators can be properly accounted for by specifying that the syntactic category Assn (which can intuitively be thought of as designating expressions that have their own assertoric forces) is in principle not embeddable. ${ }^{16}$

\footnotetext{
${ }^{15}$ As illustrated here, our analysis does not keep the at-issue and CI meanings separate in the final (i.e. Lowered) translation of the sentence. Should this treatment turn out to be inadequate, one way to retain the distinction would be to replace the boolean conjunction in the CI dimension in the lexical entries for CI triggers like (16) to an operator that produces an ordered pair, whose first element is the CI that the expression triggers and whose second element is the place holder [ ] in which the at-issue content is ultimately plugged in. In what follows, we keep the simplifying assumption introduced in the text for expository ease.

${ }^{16}$ The embedding of CIs by propositional attitude predicates (which is known to be possible at least in certain circumstances; cf. footnote 1) can be treated by allowing attitude predicates to optionally embed Assn. In this paper, we will not deal with the issue of what exactly are the (pragmatic) licensing conditions for such cases. For some discussions, see the references cited in footnote 1.
} 
In sum, the proposed continuation-based semantics for CIs allows us to handle the syntactic and semantic composition of at-issue and CI meanings in terms of the fully general mechanism of continuations: the only additional assumptions involved for the treatment of CIs are the distinct syntactic category Assn and the corresponding Assertion rule (19), which are introduced for the purpose of capturing the projective behavior of CIs: CIs do not interact with at-issue meanings in the semantic composition and their licensing contexts are significantly more restricted than other expressions (such as quantifiers) that can similarly be treated in the scheme of delayed evaluation. ${ }^{17}$

Thus, as far as typical CI triggers like parentheticals are concerned, our system has equal empirical coverage as Potts's original CI logic and the formal system involved is arguably simpler and more general—note in particular that, unlike Potts's $\mathscr{L}_{\mathrm{CI}}$, neither a type distinction nor special composition rules are needed in our system; furthermore, since our system calculates both kinds of meanings fully compositionally, the representational device called 'parsetree' is entirely eliminated as well. What, then, about the case of 'mixed' CI triggers represented by Japanese benefactives, which we know pose an empirical problem for Potts's $\mathscr{L}_{\mathrm{CI}}$ ? In the next subsection, we show that such 'mixed' CI triggers also lend themselves to a straightforward treatment in our continuation-based analysis.

\subsubsection{Japanese benefactives}

An immediate (and pleasant) consequence of giving up the type distinction between CIs and at-issue meanings (which characterizes Potts's $\mathscr{L}_{\mathrm{CI}}$ ) is that, unlike $\mathscr{L}_{\mathrm{CI}}$, our continuation-based system does not entail the problematic generalization that there can be no 'mixed' type CI contributors. Thus, an analysis of the Japanese benefactive is straightforward, to which we now turn.

The key component of our analysis is the lexical meaning of the benefactive verb morau:

$$
\left\langle\text { morau; }\left(\mathrm{NP}_{n} \backslash \mathrm{S}\right) \backslash \mathrm{NP}_{d} \backslash \mathrm{NP}_{n} \backslash \frac{\operatorname{Assn} \mid \text { Assn }}{\mathrm{S}} ; \lambda f \lambda x \lambda y \cdot \frac{\operatorname{benef}(y, f(x)) \wedge[]}{\operatorname{invlvd}(y, f(x))}\right\rangle
$$

In (21), the verb morau is specified to subcategorize for an embedded VP (i.e. $\mathrm{NP}_{n} \backslash \mathrm{S}$ ), dative and nominative arguments, and to give back a continuized meaning in which it makes meaning contributions at both the at-issue level and the CI level. Specifically, at the at-issue level, it adds the meaning that the matrix subject is 'involved' (in some way or other) in the event described by the embedded predicate; at the CI level, it contributes a benefactive meaning that states that the

\footnotetext{
${ }^{17}$ From this, it should be clear that treating CIs in terms of continuations along with other phenomena such as quantification does not immediately entail that we are conflating different linguistic phenomena as a monolithic class; differences between different kinds of delayed evaluation in natural language can be adequately accounted for by introducing a small number of phenomenon-specific assumptions. We speculate that other such differences (e.g. the fact that quantifiers but not CIs obey scope islands) can similarly be accounted for. Of course, much work is left for future research in working out the relevant details.
} 
event described by the embedded predicate is a beneficial state of affairs for the matrix subject. Note that this contrasts with the case of English parentheticals discussed above, where the at-issue meaning of the relative clause is simply an identity function, having no substantial meaning contribution.

The derivation for (9) is given in (22).

$$
\begin{aligned}
& \text { (22) }
\end{aligned}
$$

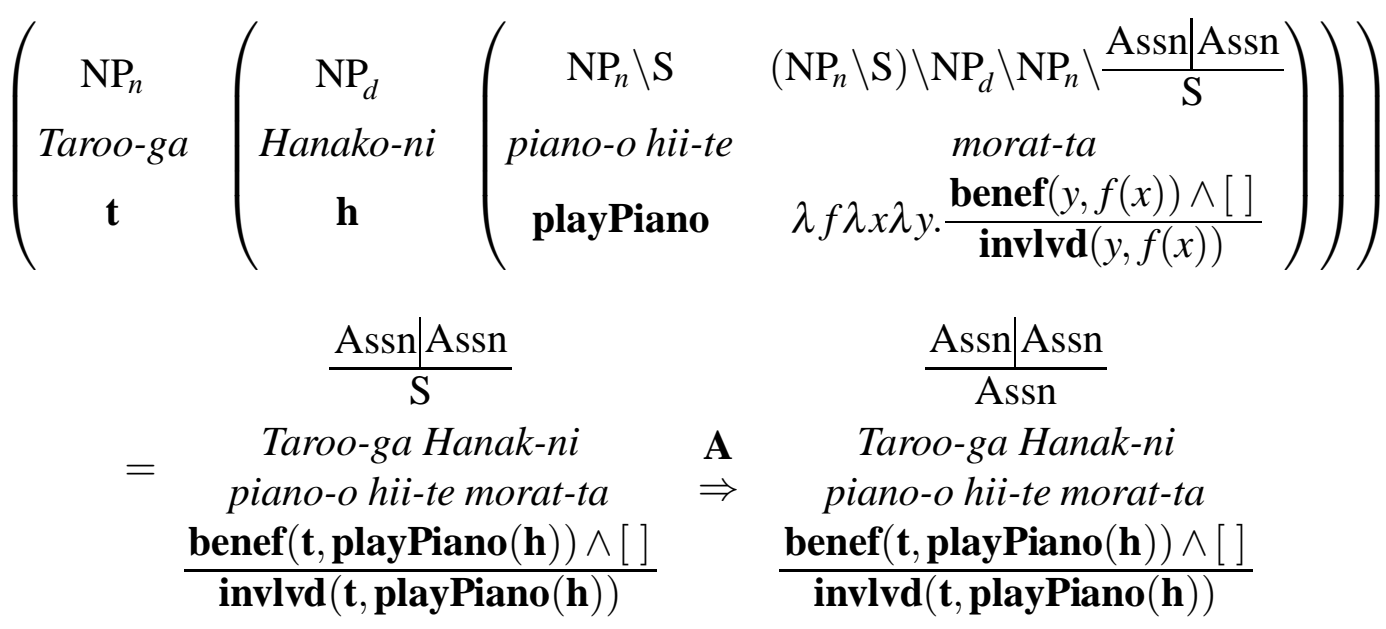

$$
\begin{aligned}
& \begin{array}{cc}
\text { Lower } & \text { Assn } \\
\Rightarrow & \text { Taroo-ga Hanak-ni piano-o hii-te morat-ta } \\
& \text { benef }(\mathbf{t}, \text { playPiano }(\mathbf{h})) \wedge \text { invlvd }(\mathbf{t}, \text { playPiano }(\mathbf{h}))
\end{array}
\end{aligned}
$$

The verb morat-ta (past tense of morau; we ignore tense here) combines with the three arguments it subcategorizes for by ordinary function application to generate the right $\mathrm{CI}$ and at-issue meaning associated with the sentence. Then, the rest is basically the same as in the English parenthetical example: the applications of the Assertion rule and Lower collapse the continuation tower so that both the at-issue content and the $\mathrm{CI}$ are treated as entailments of the sentence in the final translation.

To conclude, by employing the general mechanism of continuations, the proposed analysis correctly captures the projective behavior of CIs for both ordinary type and 'mixed' type CI triggers. The key difference between Potts's original CI logic and the proposed reformulation in terms of continuations is that the latter does away with the semantic type distinction between at-issue and CI meanings of the former, resulting in a uniform analysis of different kinds of CI triggers.

\section{Elaborating the analysis of benefactives}

We now sketch some modifications to the analysis of Japanese benefactives presented above in order to capture some further empirical facts properly. In view of a wider range of empirical data, the analysis in section 4 turns out to be too simple in two ways. First, the current analysis allows binding across CI/at-issue dimensions despite the fact that this is empirically impossible (this fact is consistent 
with Potts's (2005) generalization about the binding 'problem' of CIs). ${ }^{18}$ Second, it is not straightforward how recursive embedding of benefactive predicates (as in examples like (24) below) can be accounted for in the current analysis.

To overcome these problems, we revise the entry for morau as in (23).

$$
\left\langle\operatorname{morau} ;\left(\mathrm{NP}_{n} \backslash \mathrm{S}\right) \backslash \frac{\operatorname{Assn} \mid \text { Assn }}{\mathrm{NP}_{d} \backslash \mathrm{NP}_{n} \backslash \mathrm{S}} ; \lambda f . \frac{\forall z, w[[z, w \in \mathbf{C} \wedge \mathbf{i n v}(w, f(z))] \rightarrow \mathbf{b n f}(w, f(z))] \wedge[]}{\lambda x \lambda y . \mathbf{i n v}(y, f(x))}\right\rangle
$$

The point here is that the variables $x$ and $y$ now don't appear in the CI dimension so that it is no longer possible to bind individual variables across dimensions. Instead, the CI says that, for all of the individuals $z$ and $w$ that are contextually salient (i.e. $z, w \in \mathbf{C}$ ) and for which $\operatorname{invlvd}(w, f(z))$ is true, $f(z)$ is a beneficial state of affairs for $w$. With this entry, (9) ends up conventionally implicating that, whoever (contextually salient) person happened to be involved in some other (contextually salient) person's playing the piano, that latter's doing so was beneficial for the former. Combined with the at-issue assertion that Taro was involved in Hanako's playing the piano, the sentence effectively ends up implicating that Hanako's playing the piano was beneficial for Taro.

This revision also enables a straightforward treatment of the other problem, namely, the treatment of recursive embedding. Consider the following:

\section{Taroo-wa Hanako-ni [Jiroo-ni [hon-o yon-de age-te] morat-ta]. Taro-TOP Hanako-DAT Jiro-DAT book-ACC read BENEF BENEF-PAST}

'Taro had Hanako read a book for Jiro.'

Here, the object-oriented benefactive verb ageru is embedded under the subjectoriented benefactive verb morau. The sentence expresses the proposition given in

\footnotetext{
${ }^{18}$ Apparent cases of cross-dimensional binding like (i) should be treated as cases where the CI is predicated of some contextually salient (set of) individual(s) as in the reformulated analysis in the text (this is in line with Potts's (2005) solution for similar cases involving supplements):

(i) a. Taroo-wa dono gakusei-ni-mo syoko-no seiri-o tetudat-te morat-ta. Taro-TOP every student-DAT archive-GEN rearrangement-ACC help BENEF-PAST 'Taro had every student help with the rearrangement of the archive for him.'

b. Dono kyoozyu-mo Hanako-ni ofisu-no seiri-o tetudat-te morat-ta. every professor Hanako-DAT office-GEN rearrangement-ACC help BENEF-PAST 'Every professor had Hanako help him/her rearrange the office.'
}

Evidence for this comes from sentences like the following, where the benefactive verb is embedded in the antecedent of a conditional:

(ii) Dono gakusei-mo peepaa-o dasi-te kure-reba, kimatu.siken-o okonaw-anai. every student paper-ACC submit BENEF-COND final.exam-ACC do-NEG

'If every student submits a paper, I'll not give a final exam.'

Since the CI content projects out of the antecedent of the conditional, having the quantifier bind the CI in sentences like (ii) would force the quantifier to scope out of the antecedent as well. This, however, would give rise to incorrect truth conditions for the sentence, since, just as in English, quantifiers cannot scope out of antecedents of conditionals in Japanese. 
the above translation and additionally has the CIs that Hanako's reading the book was beneficial for Jiro (ageru) and that it was beneficial for Taro (morau) as well.

We assume the following lexical entry for ageru (the difference between morau and ageru is which of the two nominal arguments of the benefactive verbsubject or dative object—is identified as the logical subject of the embedded verb):

$$
\left\langle\text { ageru; }\left(\mathrm{NP}_{n} \backslash \mathrm{S}\right) \backslash \frac{\mathrm{Assn} \mid \mathrm{Assn}}{\mathrm{NP}_{d} \backslash \mathrm{NP}_{n} \backslash \mathrm{S}} ; \lambda f . \frac{\forall z, w[[z, w \in \mathbf{C} \wedge \mathbf{i n v}(w, f(z))] \rightarrow \mathbf{b n f}(w, f(z))] \wedge[]}{\lambda x \lambda y \cdot \operatorname{inv}(x, f(y))}\right\rangle
$$

With the lexical entries for morau and ageru in (23) and (25), the analysis for (24) is straightforward. The derivation goes as follows. First, the embedded VP Jiroo-ni hon-o yon-de age-te is of syntactic category $\frac{\mathrm{Assn} \mid \mathrm{Assn}}{\mathrm{NP}_{n} \backslash \mathrm{S}}$, and has the semantics:

$$
\frac{\forall z, w[[z, w \in \mathbf{C} \wedge \mathbf{i n v}(w, \mathbf{r}-\mathbf{b}(z))] \rightarrow \mathbf{b n f}(w, \mathbf{r}-\mathbf{b}(z))] \wedge[]}{\lambda y \cdot \mathbf{i n v}(\mathbf{j}, \mathbf{r}-\mathbf{b}(y))}
$$

To combine with this embedded VP, the matrix verb morat-ta needs to be Lifted to

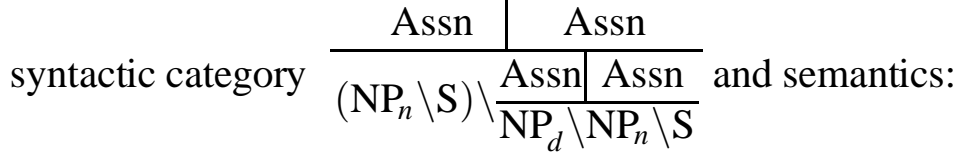

$$
\frac{[]}{\lambda f . \frac{\forall z, w[[z, w \in \mathbf{C} \wedge \operatorname{inv}(w, f(z))] \rightarrow \mathbf{b n f}(w, f(z))] \wedge[]}{\lambda x \lambda y \cdot \operatorname{inv}(y, f(x))}}
$$

Combining (26) and (27) yields the following semantics for the whole VP:

$$
\frac{\frac{\forall z, w[[z, w \in \mathbf{C} \wedge \mathbf{i n v}(w, \mathbf{r}-\mathbf{b}(z))] \rightarrow \mathbf{b n f}(w, \mathbf{r}-\mathbf{b}(z))] \wedge[]}{\forall z, w[[z, w \in \mathbf{C} \wedge \mathbf{i n v}(w, \mathbf{i n v}(\mathbf{j}, \mathbf{r}-\mathbf{b}(z)))] \rightarrow \mathbf{b n f}(w, \mathbf{i n v}(\mathbf{j}, \mathbf{r}-\mathbf{b}(z)))] \wedge[]}}{\lambda x \lambda y . \mathbf{i n v}(y, \mathbf{i n v}(\mathbf{j}, \mathbf{r}-\mathbf{b}(x)))}
$$

Application of (28) to doubly Lifted dative and nominative NPs, followed by two applications of Lower yields the desired final translation for the sentence:

$$
\begin{aligned}
& \forall z, w[[z, w \in \mathbf{C} \wedge \mathbf{i n v}(w, \mathbf{r}-\mathbf{b}(z))] \rightarrow \mathbf{b n f}(w, \mathbf{r}-\mathbf{b}(z))] \\
& \wedge \forall z, w[[z, w \in \mathbf{C} \wedge \mathbf{i n v}(w, \mathbf{i n v}(\mathbf{j}, \mathbf{r}-\mathbf{b}(z)))] \rightarrow \mathbf{b n f}(w, \mathbf{i n v}(\mathbf{j}, \mathbf{r}-\mathbf{b}(z)))] \wedge \mathbf{i n v}(\mathbf{t}, \mathbf{i n v}(\mathbf{j}, \mathbf{r}-\mathbf{b}(\mathbf{h})))
\end{aligned}
$$

Thus, with the proposed revision, cases of recursive embedding of benefactive predicates is properly accounted for. Note in particular that the availability of multiple continuation levels in the present fragment plays a crucial role in enabling a straightforward treatment of such cases.

Overall, the two empirical problems of the analysis from the previous section regarding binding across dimensions and recursive embedding of benefactive predicates can be overcome by revising the lexical entry for the benefactive verb as in (23). Although the overall picture seems somewhat complicated at first sight, note that our core proposal is still unchanged: the benefactive verb morau as (re)defined in (23) is still a 'mixed' type CI contributor and our analysis straightforwardly captures the projective behavior of its $\mathrm{CI}$ in terms of continuations. 


\section{Conclusion}

What do we learn from the continuation-based reformulation of the CI logic that we have proposed in this paper? One thing we have done is that we have verified Potts's (2005: 73) promissory remark that his $\mathscr{L}_{\mathrm{CI}}$ can be recast in non-representational terms with the help of 'semantically informed syntactic categories' (as he puts it): as is clearly visualized in the tower notation, the 'semantically informed' syntactic category Assn for the continuation level is what separates the $\mathrm{CI}$ and at-issue 'dimensions' for us. In fact, this essentially syntactic re-encoding of the original semantic type distinction is the key to achieving a fully compositional treatment of CIs: in our system, each linguistic expression has a well-defined meaning since continuized meanings can be written as ordinary lambda terms; the separation of CIs and at-issue meanings is taken care of by a syntactic category distinction, thereby eliminating representational devices within the semantic component.

More importantly, however, our reformulation is not merely a notational variant of Potts's $\mathscr{L}_{\mathrm{CI}}$. On the contrary, as we have shown, the present proposal is both conceptually and empirically more adequate than $\mathscr{L}_{\mathrm{CI}}$. The conceptual advantage lies in the fact that, in our system, CIs are treated as just a special case of delayed evaluation, which abounds in natural language. Thus, unlike Potts's original $\mathscr{L}_{\mathrm{CI}}$, our proposal does not involve any radical revamping of the fundamental architecture of the semantic theory solely for the purpose of treating CIs; rather, it treats all relevant cases of CI triggers by means of a fully general mechanism of continuations, a device that is independently needed within a formal (and fully compositional) theory of semantics. The empirical advantage is that this conceptual simplification has an immediate consequence that the problematic prediction made by the original $\mathscr{L}_{\mathrm{CI}}$ (i.e. that 'mixed' type CI triggers do not exist) simply goes away. We have demonstrated the merit of this consequence by formulating an explicit analysis of one example of a 'mixed' type CI trigger, namely, the Japanese benefactive predicate morau, in our system.

\section{References}

Amaral, Patricia, Craige Roberts, and E. Allyn Smith: 2007, 'Review of The Logic of Conventional Implicatures by Chris Potts', Linguistics and Philosophy 30, 707-749.

Bach, Kent: 2006, 'Review of Christopher Potts, The Logic of Conventional Implicatures', Journal of Linguistics 42, 490-495.

Barker, Chris: 2002, 'Continuations and the Nature of Quantification', Natural Language Semantics 10, 211-242.

Barker, Chris: 2004, 'Continuations in Natural Language', in H. Thielecke (ed.), Proceedings of the Fourth ACM SIGPLAN Continuations Workshop (CW'04), 1-11.

Barker, Chris: 2007, 'Parasitic Scope', Linguistics and Philosophy 30, 407-444. 
Barker, Chris: 2009, 'Reconstruction as Delayed Evaluation', in E. Hinrichs and J. Nerbonne (eds.), Theory and Evidence in Semantics. CSLI Publications, Stanford.

Barker, Chris and Chung-chieh Shan: 2008, 'Donkey Anaphora is In-Scope Binding', Semantics and Pragmatics 1, 1-42.

Barker, Chris and Chung-chieh Shan: 2006, 'Types as Graphs: Continuations in Type Logical Grammar', Journal of Logic, Language and Information 15, 331-370.

Cooper, Robin: 1983, Quantification and Syntactic Theory (Synthese Language Library - Text and Studies in Linguistics and Philosophy 21). D. Reidel Publishing Company, Dordrecht, Boston and London.

Harris, Jesse A. and Christopher Potts: to appear, 'Predicting Perspectival Orientation for Appositives', in Proceedings of CLS 45. Chicago Linguistic Society.

Horn, Lawrence R.: 2007, 'Toward a Fregean pragmatics: Voraussetzung, Nebengedanke, Andeutung', in J. Almog, J. Perry, and H. Wettstein (eds.), Explorations in Pragmatics: Linguistic, Cognitive and Intercultural Aspects, 39-69. Mouton de Gruyter, Oxford.

McCready, Eric: 2009, 'Varieties of Conventional Implicature: Evidence from Japanese'. Ms., Aoyama Gakuin University.

McCready, Eric and Magdalena Schwager: 2009, 'Intensifiers'. Paper presented at the Workshop on Expressives and Other Kinds of Non-truth-conditional Meaning, University of Osnabrück, March, 2009.

Morzycki, Marcin: 2008, 'Nonrestrictive Modifiers in Nonparenthetical Positions', in C. Kennedy and L. McNally (eds.), Adjectives and Adverbs: Syntax, Semantics and Discourse, 101-122. Oxford University Press, Oxford.

Partee, Barbara and Mats Rooth: 1983, 'Generalized Quantifiers and Type Ambiguity', in R. Bäuerle, C. Schwarze, and A. von Stechow (eds.), Meaning, Use, and Interpretation of Language, 361-383. Walter de Gruyter, Berlin.

Partee, Barbara H.: 1986, 'Noun Phrase Interpretation and Type-Shifting Principles', in J. Groenendijk, D. de Jongh, and M. Stokhof (eds.), Studies in Discourse Representation Theory and the Theory of Generalized Quantifiers, 115-143. Foris Publications, Dordrecht.

Plotkin, Gordon: 1975, 'Call-by-name, Call-by-value and the Lambda-calculus', Theoretical Computer Science 1, 125-159.

Potts, Christopher: 2005, The Logic of Conventional Implicatures. Oxford: Oxford University Press.

Reynolds, John C.: 1993, 'The Discoveries of Continuations', Lisp and Symbolic Computation 6, 233-248.

Sawada, Osamu: to appear, 'Modes of Scalar Reversal in Japanese', in Japanese/Korean Linguistics, Vol. 17. CSLI Publications, Stanford.

Shan, Chung-chieh and Chris Barker: 2006, 'Explaining Crossover and Superiority as Left-to-Right Evaluation', Linguistics and Philosophy 29, 91-134.

Wang, Linton, Brian Reese, and Eric McCready: 2005, 'The Projection Problem of Nominal Appositives', Snippets 10, 13-14. 